\title{
Lidar design and control tools for long-term operations
}

\author{
P. Keckhut
}

LATMOS-IPSL, University of Versailles Saint-Quentin, Guyancourt, France

\begin{abstract}
Rayleigh lidar allows to derived temperature from 30 to $80 \mathrm{~km}$. More than 30 years of continuous measurements have been performed at Observatory of HauteProvence providing a long term cooling. Along the years of operations, the technique has been improved. The design of the lidar is described here. The emission and reception benefit for advance solutions of coupled lasers or mirrors with optical fibbers. Alignment and synchronization are also described, while noise estimates correspond to an issue when upper mesosphere want to be investigated.
\end{abstract}

\section{Introduction}

Rayleigh lidars that provide temperature retrievals using molecular scattering [1-3], have been operated in a routine basis since the late 1970's at Observatory of Haute-Provence (OHP), France, and since 1991 from several observatories in the world in the frame work of the Network for the Detection of Stratospheric Changes [4] now renamed Network for the Detection of Atmospheric Composition Changes (NDACC). Long-term operations required to avoid time discontinuities and then to develop to the most robust and reliable techniques. This article provides technical choice for the lidar design implemented along the 30-years of continuous operations at OHP, and the different other more recent projects driven by the Laboratory of Atmospheres, Mediums and Observations from Space (LATMOS).

\section{General design of Rayleigh lidars}

A pulse light is sent vertically into the atmosphere (Fig. 1). The light scattered by the molecules is collected using a telescope. Photons are detected with a photomultiplier after the background light being filtered out to keep only the laser light scattered by the molecules.

The main error is associated with the number of photons detected. To reduce this statistical noise as described in [3], the system should be able to collect the largest number of photon. In the lidar equation (3), the main factors are: the laser energy, the scattering efficiency (decided by the wavelength used), the atmospheric transmission, the system efficiency and the detector. The preferred spectral domain is located from the near Ultra-Violet to the visible wavelengths (Fig. 2(b)). The Rayleigh scattering efficiency varies as the inverse of the fourth power of the wavelength in favor to the short wavelengths, while the atmospheric attenuation is the largest in the Ultra-Violet range. Also the detector efficiencies fall drastically in the infrared domain. The transmission of the optics are generally better in the visible infra-red domain compared to the Ultra-Violet, but exhibits differences sufficiently small and so are not a constrain factor.

This is an Open Access article distributed under the terms of the Creative Commons Attribution-Noncommercial License 3.0, which permits unrestricted use, distribution, and reproduction in any noncommercial medium, provided the original work is properly cited. 


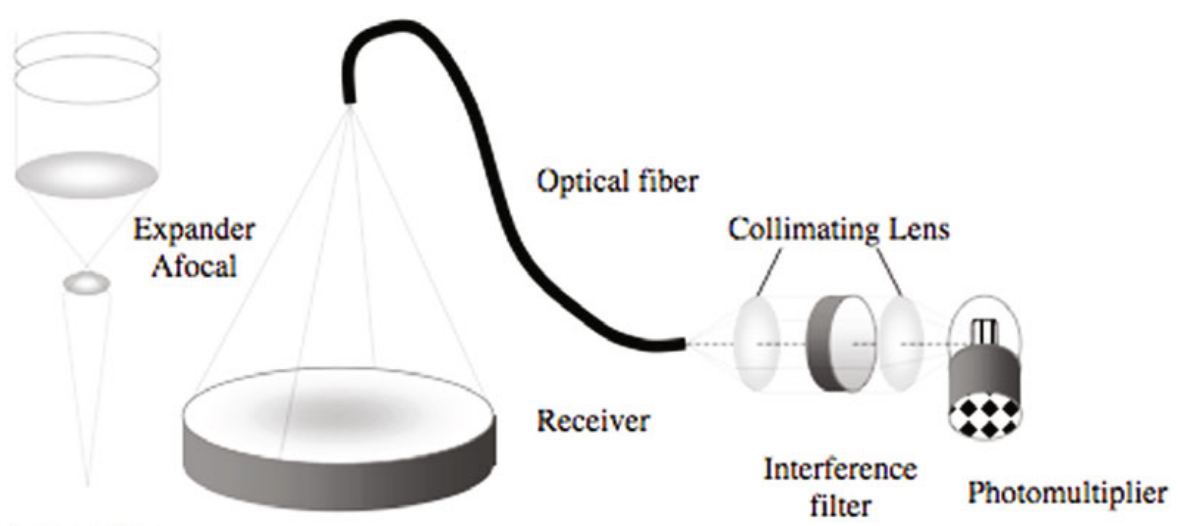

Laser pulse

Fig. 1. Basic schematic of the Rayleigh lidar.

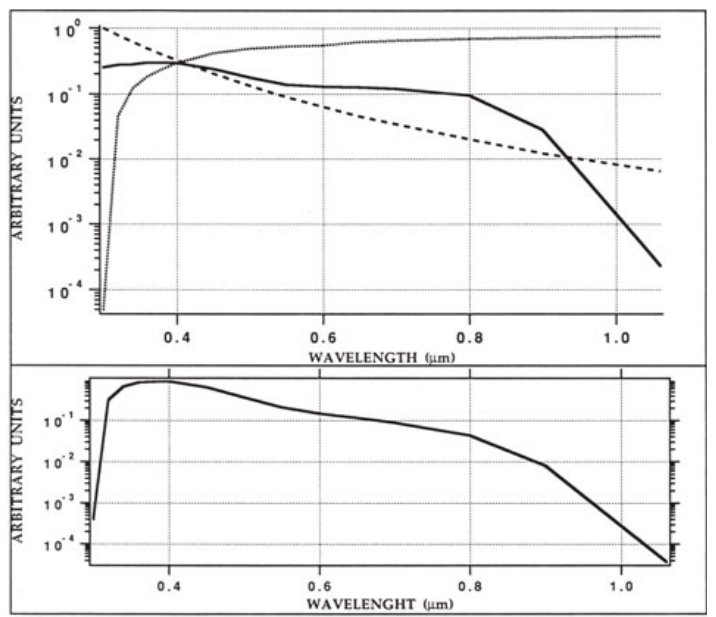

Fig. 2. Efficiency as a function of the wavelength of the Rayleigh scattering (dash line), the atmospheric transmission (grey line) and the detector efficiency (dark line) are represented in the top panel. Bottom panel corresponds to the final system efficiency given by the product of the 3 above parameters.

In this spectral domain, several powered laser sources are available [5]. The most popular one was the Ruby laser at $694 \mathrm{~nm}$ that can provide large pulse energy but moderate repetition rate $(1 \mathrm{~Hz})$, while at present time the Neodyme:Yag lasers, respectively frequency doubled $(532 \mathrm{~nm})$ or triples $(355 \mathrm{~nm})$ are the most efficient ones in providing the largest energy and larger pulse rate $(10-50 \mathrm{~Hz})$. Frequency double Titane-Sapphire $(395 \mathrm{~nm})$ and Alexandrite $(377 \mathrm{~nm})$ are alternated laser sources but provide less energy. The pulse energy is not the main parameter but the laser power that is the product of the pulse rate and the energy per pulse as soon as there is no time overlap between two successive echoes. The time required for the light to get up vertically to the highest altitudes of the atmosphere where significant scattering signals can be detected (around $100 \mathrm{~km}$ ) including the time to return back is around 0,7 millisecond, and if the time for noise evaluation is also considered the total correspond to one millisecond. The maximum laser pulse rate can be as high as $1 \mathrm{kHz}$.

In the UV-visible spectral domain where the scattering and detection efficiency is the largest, the chosen wavelength depends of the power of the pulse light available. In this domain solid-state laser are available and contribute to the robustness of the Rayleigh lidar technique by itself.

The maximum altitude range is associated with the number of photons and then the noise need to be considered as a limited factor. To perform the best measurement the noise need to be reduced at its minimum. Noise is due to several sources: sky background, dark current and induced noise. 
The parasite light from the sky background is considerably limited by dark time operation. However in the visible domain, even during dark time it remains the largest source of noise. An interference filter providing a bandwidth between $1-10 \mathrm{~nm}$ is then placed before the detector. Also narrow fields of view for the telescope used as collector are choose to reduce the portion of the sky seen by the telescope and then the noise. This is quite efficient for noise reduction but render the alignment between laser emission and telescope collection even more complicated. The dark current can also be reduced in using cooling systems based on Peltier effects or cold water flow. The last source of noise for photomultipliers is the signal itself. The detector noise is very sensitive to the initial burst. If this can be avoided, a background level of 10-100 count/second can then be achieved.

To calculated the range for a given lidar systems, the accuracy at the top altitude $z_{0}$ need to be fixed; Usually a value a $10-15 \%$ is used corresponding to the natural variability and then based on the statistical error formula:

$$
\frac{\Delta n\left(z_{0}\right)}{n\left(z_{0}\right)}=0.15=\frac{\sqrt{\left(N\left(z_{0}\right)+B_{P M}\right)}}{N\left(z_{0}\right)} .
$$

The signal should be larger than:

$$
N\left(z_{0}\right)=\frac{\sqrt{\left.B_{P M}\right)}}{0.15} .
$$

For a given time and vertical average, the signal can be theoretically estimated using the full lidar formula. Because the main parameters for a lidar is the surface telescope area and the laser power, another possibility to estimate the altitude range or the accuracy, consists in comparing a new system with an existing systems. The error can be expressed as:

$$
\frac{\Delta n(z)}{n(z)}=K \frac{z^{2} \sqrt{B_{P M}}}{\sqrt{N_{0} S_{T} n(z)}} .
$$

With $\mathrm{N}_{0} \mathrm{~S}_{\mathrm{T}}$ can be named as the lidar power, and $\mathrm{K}$ a constant value related to the lidar efficiency. Then if the measurement characteristics for a system at a level 1 is known, the effect of an increase of the lidar power can be estimated on a the level 2 of the same lidar system. This is particularly interesting to calculate the expected factor of improvement $F I$ when laser or telescope area are modify.

$$
F I=\sqrt{\frac{N_{2} S_{T 2}}{N_{1} S_{T 1}}} .
$$

If we considered the atmospheric density decreasing exponentially with a scale height of $\mathrm{H}$ then the range improvement can be estimated as a first approximation as:

$$
z_{2}-z_{1}=-H . \operatorname{Ln}\left[\frac{N_{1} S_{T 1}}{N_{2} S_{T 2}}\right] .
$$

\section{Optical design}

The optical design is driven by two main objectives: increasing the number of photons and decreasing the background noise according to Eq. (1). The number of collected photons is directly proportional to the laser energy send in the atmosphere, the scattering efficiency associated with the wavelength, the overall optical transmission and the detector efficiency. The noise depends primarily to the light coming from the sky background even during nighttime. The reduction of the level of noise is then performed in decreasing as soon as possible the field of view of the telescope and then the portion of the sky seen by the detectors. Also the bandwidth of the filter can be reduced to the bandwidth of the scattered light. During nighttime, the background noise can be reducing sufficiently to be negligible compared to the dark current of the detector. 


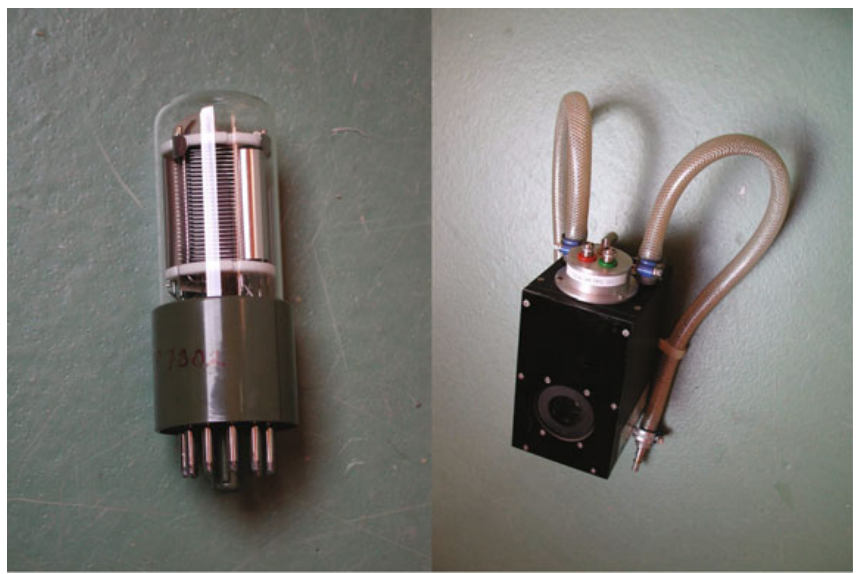

Fig. 3. Picture of the Photomultiplier side-window (left) and the mount including the Peltier effect and the watercooling system (right).

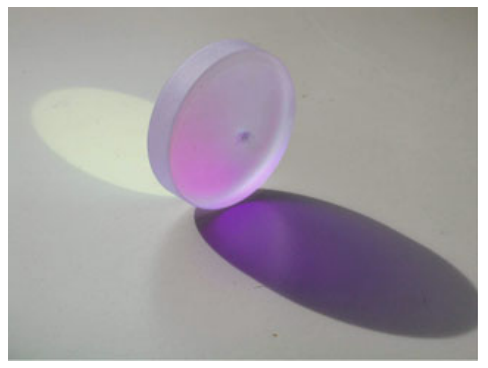

Fig. 4. Optical device used for laser beam transfer before to be send in the atmosphere (left). The purple color in transmission denotes the complementary color (green). A hint on the surface can be noted.

The number of photons collected depend of the laser power and so the chosen wavelength as shown in Fig. 2. The size of the laser is directly related to the photon collected. While the mean power correspond to several watt, the pulse duration is only $10-20 \mathrm{~ns}$ and then the instantaneous energy is quite high even with $10-100 \mathrm{~Hz}$ laser repetition rate. The beam deviation from the laser to the zenith direction requires using mirror that exhibit a high degree of reflection. If not, the energy that is absorbed is large enough to destroy the substrate. Multi-layer coating are used to insure reflection coefficient of $99,99 \%$ at the required angle (Fig. 4). Laser power can be increase by combining several laser sources. When 2 lasers are combined the polarization can be used to couple laser bean in the same direction. When more laser beams have to be used, a geometrical combination can be implemented.

While the cost of the astronomical telescopes is not linearly related with its surface [7] and because lidar do not require telescope to perform image reconstruction, the use of mosaic mirrors were quite adapted to such application (Fig. 5). To achieve this challenge, optical fibbers were used to connect the different surface areas in one final beam.

The mirror mosaics, as light collector, associated with optical fibbers offer the opportunity to increase, at a reduce cost, the collector surface, to provide a small signal attenuation, and present many other advantages for lidar operations:

- It insures a mechanic decoupling between emission and reception that means that as soon as fibbers are fixed at the focus plan of the telescope and in the other side at the optical box (including filters and detector), the both systems can move without any effect.

- It insures a thermal decoupling because temperature in the receiver area can decrease during night that can have impact on the optical filtering box.

- It allows a progressive implementation or increase of the collector surface. 

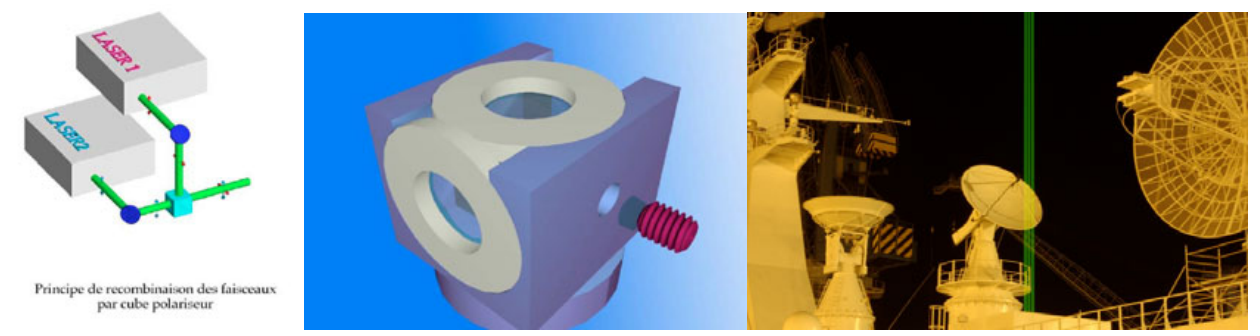

Fig. 5. Laser coupling (left) with polarizer cube (middle). Lidar on Monge ship used the geometrical combination of 3 laser beams (right) that are send vertically and can be identified visually.

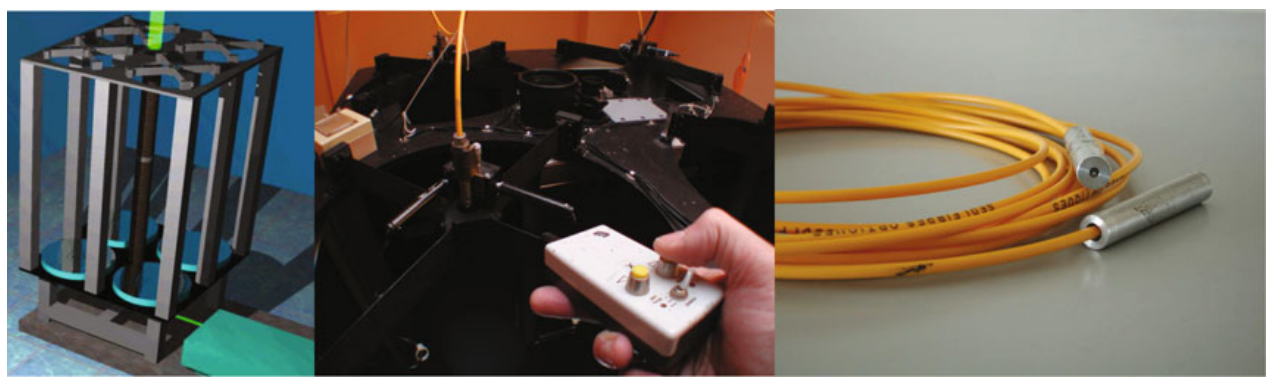

Fig. 6. Mosaic of telescope (left), zoom on the optical fibber and the X-Y displacement motorized system (middle), and an optical fibber with mounts at each edge (right).

Focal telescope should be large enough to focus the light into the optical fibber within the accepting cone of the optical fibber. If this restriction is insured, then the light will transit through the optical fibber with a limited attenuation and come out with the same cone that the one used at the entry of the fibber.

The field of view $\alpha$ of each individual telescope is related to the diameter of the optical fibber $d$ and the focal of the telescope $f$ by the following formula:

$$
\alpha=d / f .
$$

The lower limit depends of the laser divergence and the altitude range (defocalization effect) and the sensitivity accepted to insure the alignment between emission and reception. Such system has been implemented with 8 telescopes of $50 \mathrm{~cm}$ diameter for a lidar dedicated to the mesosphere [2] providing a total surface of 1,6 meters. This system presents however sometime some limitations when other simultaneous measurements are performed simultaneously: optical fibber fluorescence by Rayleigh scattering [8] or when field of view need to be changed. Multi-optical fibbers-systems have been proposed but those remain complex to implement [9]. No optical systems are used to interface the optical fibber either on the telescope and either on the optical box. The faces of the fibber are polished and inserted into a metallic mount that protect each side and help in fixing the optical fibber.

One of the main issues with narrow field of view is the alignment between emission and reception. To reduce the laser divergence, an afocal optical system is used to reduce the initial laser divergence to around $10^{-4}$ in using a reduction factor of $10-15$ that also increase the section of the emission pulse by the same factor and reduce slightly the eye risks. The divergence cannot be infinitely reduced [10], because small-scale inhomogeneities may cause significant reduction in laser brightness, resulting to an overall increase of the angular divergence.

The photons scattered from high altitude and collected, converge to the focus plane and exhibit the smallest diameter that can be easily calculated according to laser divergence and focal length of the telescope. However as far as lower altitudes are considered, the photon converge in a plane behind the focal plane and thus the intercepted diameter of the beam is larger and also displaced 


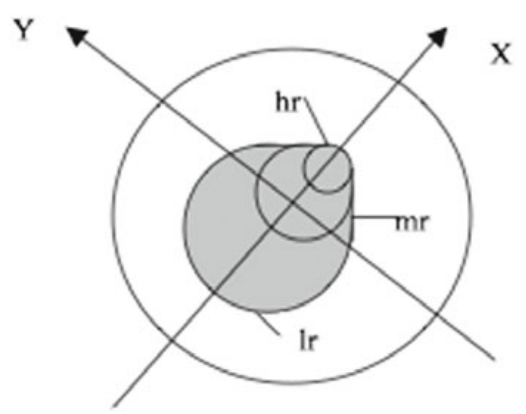

Fig. 7. Schematic of the light distribution in the focus plane of the telescope according to altitude.

according to the vertical axis of the telescope if the emission is not coaxial (Fig. 6). To compensate these both effects the diameter of the optical fibber need to be larger. The knowledge of the light distribution after the telescope is helpful for insuring alignment.

To found the good alignment between emission and reception, preliminary laboratory setup can be performed. Either laser beam or telescope can be adjusted, however in case of multi-mirror receiver, the laser direction should be chosen as the reference. Telescopes are not displaced, but optical fibbers are move on the focal plane of the telescopes. Few micrometers range is necessary to insured alignment. Manual mechanical systems can be used but they can be interface with small motors and the drive by the computer in case of automatic alignment process (Fig. 6). Corner cube retro-reflector can also be used to improve on site the pre-setting. Corner Cube system are designed to reflect any ray or beam entering one face, regardless of the orientation of the device, back onto itself. Such a system can be mount above the afocal to insure preliminary alignment adjustment. However, those systems are not enough accurate and the signal is observed with an oscilloscope to improve the final setting. One difficulties is that the operator tends to maximize the largest signal which is the one coming from the lowest altitude, then the highest part of the signal should also be investigate to be sure that the full range of the lidar signal in the concerned range is entering the optical fibber. The experience shows that below a ratio of 2-3 between the expected size of the light distribution in the face of the fibber and the diameter of the fibber, alignment are nearly impossible to insure. Automatic systems are developed to perform these operations. Lidar signal are acquired and one specific level, corresponding to the one where signal should be optimized and where signal are sufficiently large. Integration over several shots are often required to obtained a signal accurate enough to detect changes associated to alignment effects. Usually both sides of the image need to be detected to found and optimal position of the fibber. Whatever the method, very stable atmospheric conditions are required to attribute signal changes only to alignment effects rather than atmospheric attenuations.

When different channels, with different field of view, are used. The ratio between the both channels, in the altitude range where both signals are valid, should indicate a constant value if the alignment is correctly achieved. When alignment is not perfectly insured, the channel having the smallest field of view presents the largest probability to have miss-alignment that will induce the largest effect at lower altitude. The defocalization as well as the parallax effects, induces an effect that varies at the first order as the inverse of the altitude.

On the optical box a limited number of devices are required. Photons coming out of the optical fibber pass through a lens to provide a collimated beam that can be filter out (narrow filters operated efficiently for a given incident angle) with a filter. Usually on interference filter (multi-coating filter) is used to remove the background light (Fig. 1). Then another lens is required to focus light on the detector window. The filter reduces the transmission and reduces noise, which are two antagonist effects. To decide which filters is adequate, the following formula can be derived from equation 1 with $\Delta_{\mathrm{F}}$ being the bandwidth of the filter and $\mathrm{T}_{\mathrm{F}}$ the peak transmission:

$$
\frac{\Delta n\left(z_{0}\right)}{n\left(z_{0}\right)}=\frac{\sqrt{B_{P M}}}{N\left(z_{0}\right)}=\frac{\sqrt{\Delta_{F}}}{T_{F}} .
$$




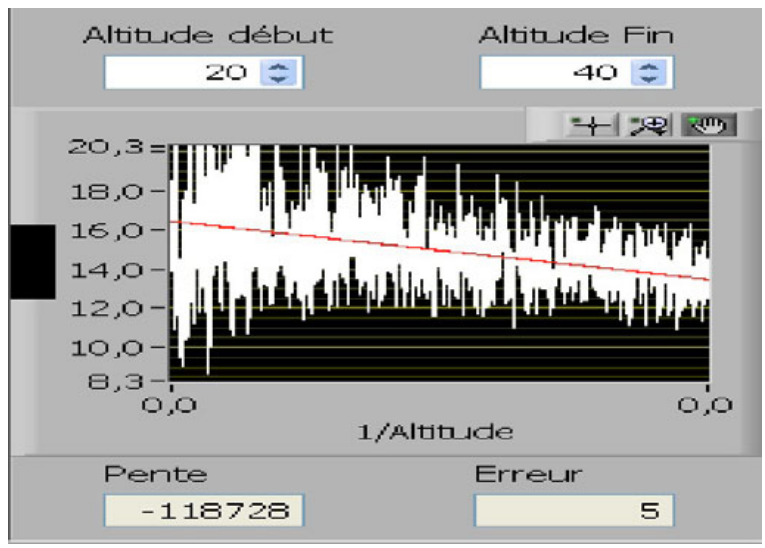

Fig. 8. Ratio between two channels having different fields of view. The slope as a function of the inverse of the altitude, instead of a constant value, indicates a non-perfect alignment.

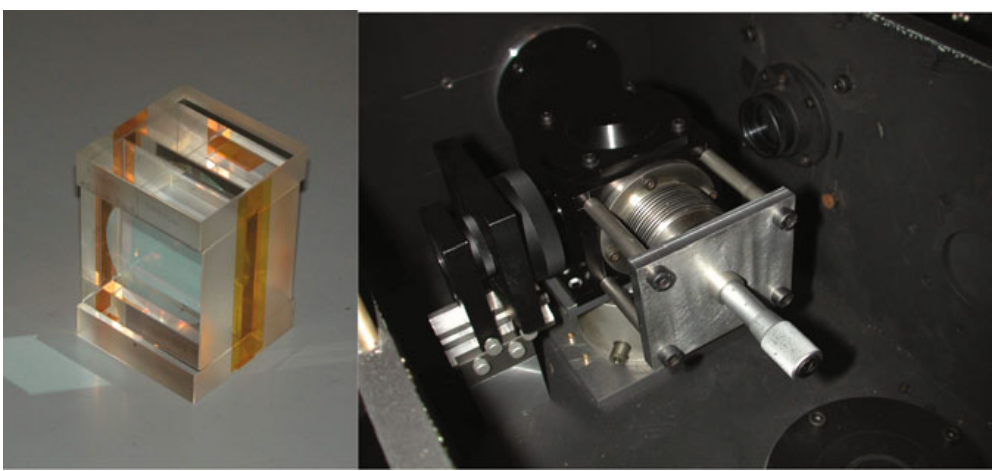

Fig. 9. Mono-bloc Fabry-Perot (left) as used for wind measurements [11] and its pressure cell (right) to adjust the spectral peak to the laser wavelength.

For daytime conditions this choice is a critical one. Fabry-Perot spectrometer (Fig. 9) that provides very narrow bandwidth is a good candidate. However, broadband filter are also required to remove noise from all the spectral peak of the spectrometer.

\section{Electronic issues}

The detection of the photon arrival is performed by a photomultiplier. Because the signal is weak the detection mode is the photo-counting. Photoelectrons lead to electric pulses that are counted during a certain interval that decide for the vertical resolution. Usually light pulses range from 20 nanoseconds to 1 microsecond corresponding to several meters to several hundred meters resolution. Each sampled echo is not save on the computer because the interface between electronic and the computer is not sufficient. Then several shots are accumulated according to a reference time corresponding to laser pulse emission. After hundreds to thousands shot integration, the information is saved on the computer. Several shot may be lost during this phase. Two method can be used to insure the synchronization between electronic and the laser. Or the electronic is the master and the laser is the slave. In that case, electronic sends a fire order to the laser and a certain time later start to count. The reverse is possible and preferred. The laser is firing at its own rhythm, and the pulse is detected using parasite scattering light on a mirror that start the counting. The main advantage of this second method is that the laser rate changes do not have any impact on the synchronization. However, when using mechanical shutter or several lasers as DIAL system do to derive temperature, this solution is not feasible. 


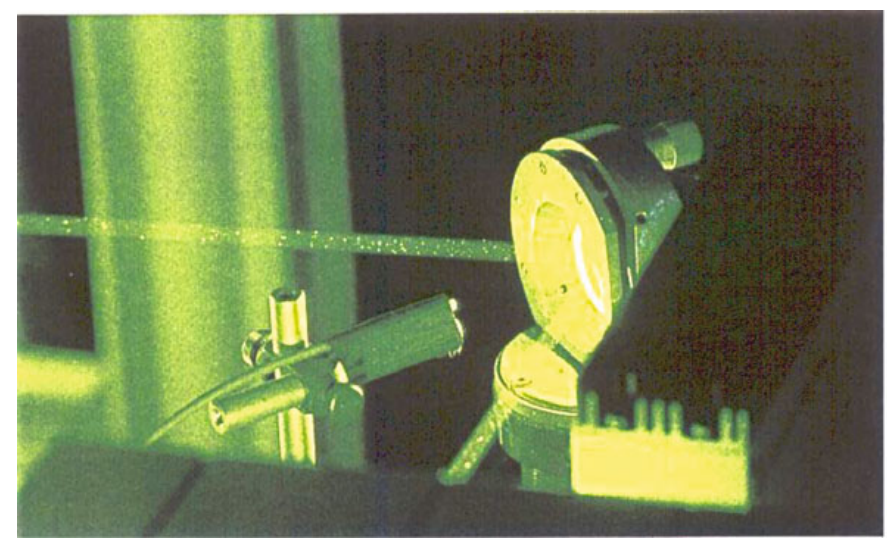

Fig. 10. Light pulse before zenith emission. Scattering is detected by an optical fibber for electronic synchronization.

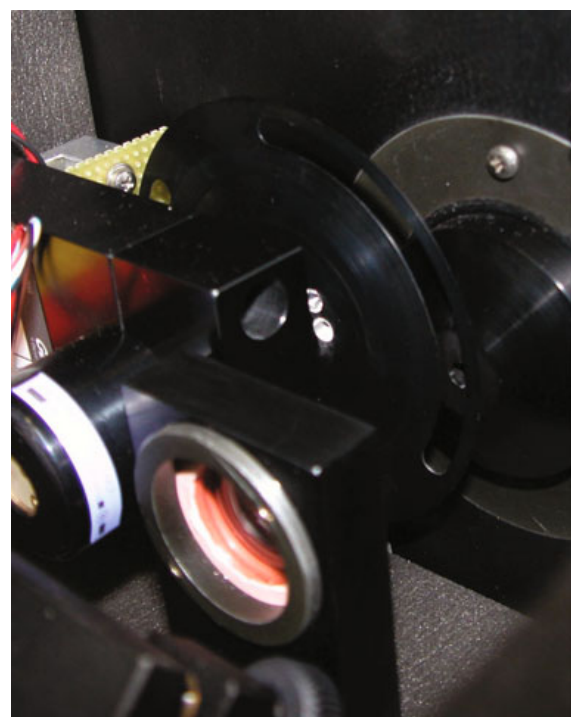

Fig. 11. Example of rotating shutter used to prevent photomultiplier against initial burst.

One of the main issues about the accuracy of the photo-counting is the quality of the pulse and their selection among the noise pulse. If pulses exhibit any after pulse, then the counting can be biased. The selection of signal pulses among noise is performed using a discriminator. The noise and signal pulse distributions often exhibit overlap and perfect separation is not always possible. An optimum compromise must be found. The photomultiplier high voltage and electronic amplifier are very sensitive and should be setup correctly according to pulse distribution. The quality of the counting can be check with constant photomultiplier illumination as the variability should be close to the theoretical value of the square of the number of counted photon.

While noise due to background sky luminance can be reduce to a negligible level, the signal induce noise is a limited factor. A photomultiplier exhibit a noise that depends of the signal. While the initial burst of the lidar signal is quite large, an induced noise is generated that evolve with time during the echo. The correction of such exponential decrease of the noise is quite challenging while both the noise and the signal decrease with time. A parabolic function is applied to quantify such noise trend because this function appears to provide more stable results than an exponential function. However the altitude range where signal fitting remains performed is an issue. To improve such estimate, expected 
signal in the noise range is evaluated in extrapolating the scattering signal from lower altitude in using a density atmospheric model. The signal, made by the difference of the raw signal minus the expected scattering from the density, allows a better estimate of the noise evolution. However, the reduction of this effect of signal-induced noise can be reduced by adequate hardware design. Because the Rayleigh temperature is derived only above $30 \mathrm{~km}$ where aerosol density is negligible, the initial signal on the photocathode of the photomultiplier can be avoided. Several solutions have been implemented: the parallax effect, exhibit a geometric field recovery than reduce signal, the rotating disc is one of the best solution to insure a full

And finally photomultiplier high voltage can be inverted to reduce the photon arrival on the photocathode. This last solution is fully electronic and present the advantage of being easily adjustable but the high voltage re-initialization introduce a transition period when signal can be biased. The high voltages commutation on several dynodes simultaneously reduces this transition effect.

The full coverage of the dynamical range from 30 to $100 \mathrm{~km}$, requires several channels with different sensitivity. Lam of glass can be used to extract before the detection a small portion of the signal to compose an extra channel for the lower altitudes. The both channels have the same field of view and do not permits to make comparisons and alignment checking. Another solution consists to have dedicated telescope with surface area fitting the altitude range expected. The filed of view of the lowest channels can be larger to take into account for defocalization effects and then can be used as reference to check alignment qualification for the upper channel having the smallest field of view to reach the highest altitudes.

\section{Conclusions}

The long-term lidar operations performed at Observatory of Haute-Provence lead to improve our knowledge of instrumental design to derive temperature profiles in the stratosphere and mesosphere. To obtain the best accuracy in the temperature retrieval, the increase of collected photons is required. To improve the accuracy as well as he experience shows that the simplest systems are the most robust. The use of mosaic of mirrors coupled with optical fibbers was a critical improvement. Alignment between emission and reception are also critical because small fields of view are used. Multi-telescope receivers are used to cover the dynamical range and to provide a possibility to evaluate the quality of the emission-reception alignment. The reduction of noise and mainly the signal-induced noise is also a challenge to improve the altitude range.

\section{References}

1. A. Hauchecorne, M.L. Chanin, Geophys. Res. Lett., 7, 565-568 (1980)

2. P. Keckhut, A. Hauchecorne, M.L. Chanin, J. Atm. Ocean. Tech., 10, 850-867 (1993)

3. P. Keckhut, Adv. Space Res., 28, 955-959 (2001)

4. M.J. Kurylo, S. Solomon, NASA report, Upper Atmosphere Research Program and NOAA Climate and Global Change Program (NASA), Washington, D.C (1990)

5. C.A. Nanni, T.S Alster, R.G. Geronemus, Dermatologic surgery, 24, 1399-1405 (1998)

6. Weber, Marvin J. Handbook of laser wavelengths, CRC Press, ISBN 0849335086 (1999)

7. G.T. van Belle, A.B. Meinel, M.P. Meinel, The scaling relationship between telescope cost and aperture size for very large telescopes, Proc. SPIE 5489, 563 (2004)

8. V. Sherlock, A. Garnier, A. Hauchecorne, P. Keckhut, Applied Optics, 38, 5838-5850 (1999)

9. J.L. Baray, J. Leveau, J. Porteneuve, G. Ancellet, P. Keckhut, F. Posny et S. Baldy, Appl. Opt., 38, 6808-6817 (1999)

10. V.D. Dubrov, R.V. Grishaev, M.D. Homenko, Yu. N. Zavalov, Laser Physics 19, 1131-1135, DOI: 10.1134/S1054660X09050429 (2009)

11. A. Garnier, M.L. Chanin, Appl. Phys. B 55, 35-40 (1992) 